\title{
Distance Based Topological Indices And Regular Graphs
}

\author{
PuruchothamaNayakiMa ${ }^{\mathrm{a}}$,Dr.Simon raj $\mathbf{F}^{\mathrm{b}}$ \\ a Assistant Professor, Dept. of Mathematics, Dhanraj Baid Jain College, Chennai. \\ ${ }^{\mathrm{b}}$ Assistant Professor, Hindustan Institute of Technology and Science, Chennai, Tamilnadu, India
}

Article History: Received: 10 November 2020; Revised 12 January 2021 Accepted: 27 January 2021; Published online: 5 April2021

\begin{abstract}
In this article, we are using the regular graph of even number of vertices and computing the distance balanced graphs. First we take a graph for satisfying regular definition and then we compute the Mostar index of that particular graph. If the Mostar index of that particular graph is zero, then the graph is said to be a distance balanced graph. So we discuss first distance balanced graph. Suppose if we delete one edge in that particular graph, that is non-regular graph, we can verify the balanced graph is whether distance balanced graph or not. We discuss and compute the Mostar index of certain regular and non-regular graphs are balanced distance or not. Finally we see few theorems are related in this topic. So in this paper, we study some distance based topological indices for regular graphs and also cubic graphs.
\end{abstract}

Keywords: Mostar index, Regular graph, Non-regular graph, Distance balanced graph, Cubic Graph

\section{Introduction}

We have graph theory, it is a branch of mathematics but at present graph theory is mostly used in chemistry and physics departments for their research studies. In chemical graph theory we have seen that graph theory plays a wonderful role for growing chemical bond connectivity, QSPR analysis and QSAR analysis. In graph theory, topological indices are widely used for computing and calculating the degree based topological indices and distance based topological indices. In degree based topological indices we have the following indices namely Balaban index, Gutman index, $\mathrm{ABC}$ index and so on. In the distance based topological indices we have the following indices namely wiener index, Mostar index, edge wiener index, szeged index and so on. We have wiener index is the most commonly index for calculating and computing the distance based topological indices for certain molecular graphs. Most of the Researchers they can take wiener index for their Research work. Most of articles are published in their wiener index method. Also wiener index is the base for our topological indices. Here in this article we are using the one of the distance based topological index namely Mostar index. In this article we prove regular graphs of even number of vertices, which are also distance balanced graphs. The definition of distance balanced graph is, the number of points (Vertices) closer to $u$ than $v$ is equal to the number of points (Vertices) closer to $v$ than $u$. That is the value of Mostar index is Zero. i.e, $M_{o}(G)=0$.

This definition is called distance balanced graph. So regular graphs of even number of vertices are also distance in balanced graphs. This result is the main result of our article. So Finally, in the article, we introduce regular graphs and to compute the Mostar index value is zero also we discuss the cubic graphs are also distance balanced graphs.

\section{Definitions and Preliminaries:}

A graph is a set of an unordered pair of vertices and edges where $V(G)=$ vertex set of $G$ and $E(G)=e d g e$ set of $\mathrm{G}$. In a graph $\mathrm{G}$, the number of lines incident with $\mathrm{V}_{\mathrm{i}}$ is said to be a degree of $\mathrm{V}_{\mathrm{i}}$ and is denoted by $\mathrm{d}_{\mathrm{G}}\left(\mathrm{V}_{\mathrm{i}}\right)$ or $\mathrm{d}\left(\mathrm{V}_{\mathrm{i}}\right)$. The distance of the graph $\mathrm{G}$ is the number of lines passing through the path is said to be a distance of a graph G. A graph is said to be a regular graph, if the minimum degree vertices of $G$ is equal to the maximum degree vertices of $\mathrm{G}$.

$$
\text { i.e., } \partial(\mathrm{G})=\Delta(\mathrm{G})=\mathrm{r}
$$

Where $\quad \partial(\mathrm{G})=$ minimum degree vertex set,

$\Delta(\mathrm{G})=$ maximum degree vertex set.

A graph is said to be non-regular graph if $\partial(\mathrm{G}) \neq \Delta(\mathrm{G})$ - also a graph is said to be a cubic graph if it is a regular graph of degree 3 .

The wiener index of a graph $\mathrm{G}$ is the sum of the distance between all pairs of vertices of $\mathrm{G}$ 
ie., $W(G)=\sum d_{G}(u, v)$

$$
(\mathrm{u}, \mathrm{v}) \underline{\mathrm{c} v}(\mathrm{G})
$$

Where $d_{G}(u, v)$ is the shortest distance between $u$ and $v$.

For trees wiener index is also defined as

$\mathrm{W}(\mathrm{G})=\sum \mathrm{n}_{\mathrm{u}} \mathrm{n}_{\mathrm{v}}$

$\mathrm{u}, \mathrm{v} € \mathrm{E}(\mathrm{G})$

Where $n_{u}$ denotes the number of points(vertices) of $G$ closer to $u$ than $v, n_{v}$ denotes the number of points(vertices) of $\mathrm{G}$ closer to $\mathrm{v}$ than $\mathrm{u}$.

The mostar index of a graph $\mathrm{G}$ is the difference between the number of vertices closer to $u$ than $v$ and the number of vertices closer to $\mathrm{v}$ than $\mathrm{u}$

$$
\text { i.e., } \quad \begin{aligned}
& \operatorname{Mo}(G)=\sum\left|n_{u}-n_{v}\right| \\
& e=u v € E(G)
\end{aligned}
$$

A graph $\mathrm{G}$ is said to be distance balanced graph, if the number of vertices closer to $\mathrm{u}$ than to $\mathrm{v}$ is equal to the number of vertices closer to $\mathrm{v}$ than to $\mathrm{u}$

i.e., $\operatorname{Mo}(\mathrm{G})=\sum\left|\mathrm{n}_{\mathrm{u}}-\mathrm{n}_{\mathrm{v}}\right|=0$

A graph is said to be distance balanced graph, if $\operatorname{Mo}(G)=0$.

\section{Distance Balanced Graphs}

\section{Example:1}

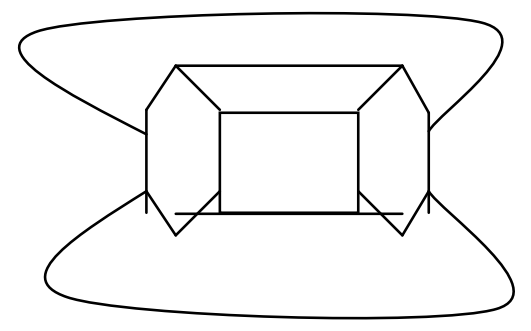

In this figure, we have the number of vertices $=12$,

The number of edges $=18$

The calculation of Mostar index value $=0$

So the above graph is a distance balanced graph and also an even number of vertices.

\section{Example: 2}

In this figure,

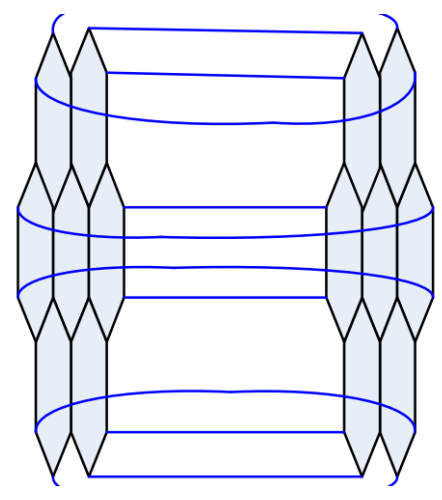

The number of vertices $=48$

The number of edges $=78$ 
The calculation of Mostar index value is Zero. So it is also a distance balanced graph.

\section{Example: 3}

In this figure,

The number of vertices $=108$

The number of edges $=166$

The calculation of Mostar index value is Zero. So it is also a distance balanced graph.

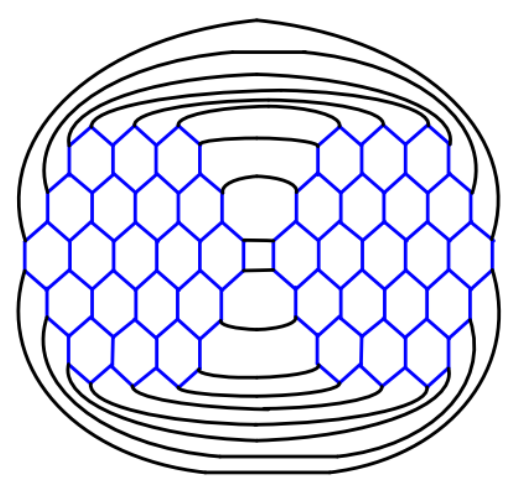

\section{Lemma:}

A graph is regular with an even number of vertices if and only if the Mostar index of G is zero.

\section{Solution:}

Let us assume, graph $\mathrm{G}$ is regular

If $\mathrm{G}$ is regular, $\partial(\mathrm{G})=\Delta(\mathrm{G})=\mathrm{r}$

In Ex1, we have $\partial(\mathrm{G})=\Delta(\mathrm{G})=3$

So, it is a distance balanced graph.

i.e., $M_{0}(G)=0$

Conversely, Suppose $M_{o}(G)=0$

That implies $\partial(\mathrm{G})=\Delta(\mathrm{G}$.

So, it is a regular graph with an even number of vertices.

\section{Theorem:}

For any regular graph $\mathrm{G}$ of even number of vertices, the cycle $\mathrm{n}$ is a distance balanced graph.

\section{Proof:}

We have to prove this theorem by using induction hypothesis method.

Let us take $\mathrm{P}(1)=$ cycle of order 1

In example 1 , we have $\mathrm{M}_{\mathrm{o}}(\mathrm{G})=0$

So, $\mathrm{P}(1)=0$

In example 2 and 3 , we have

$\mathrm{P}(2)=\mathrm{P}(3)=0$

Let us assume that the cycle of order (n-1) is true.

i.e., $P(n-1)=$ cycle of order $(n-1)=0$

To prove $\mathrm{P}(\mathrm{n})$

$P(n)=$ cycle of order $n$ 
$=$ cycle of order $(n-1)+$ cycle of order 1

$\mathrm{P}(\mathrm{n})=0$

Hence, the cycle of order $\mathrm{n}$ is a distance balanced graph.

\section{Non Distance Balanced Graphs}

\section{Lemma:}

A graph is non-regular, then it is not a distance balanced graph.

\section{Proof:}

Given graph is a non-regular graph, that

is $\partial(\mathrm{G}) \neq \Delta(\mathrm{G})$

So that reason $\mathrm{M}_{0}(\mathrm{G}) \neq 0$

Hence, it is not a distance balanced graph.

\section{Example 1:}

In this example, we see that

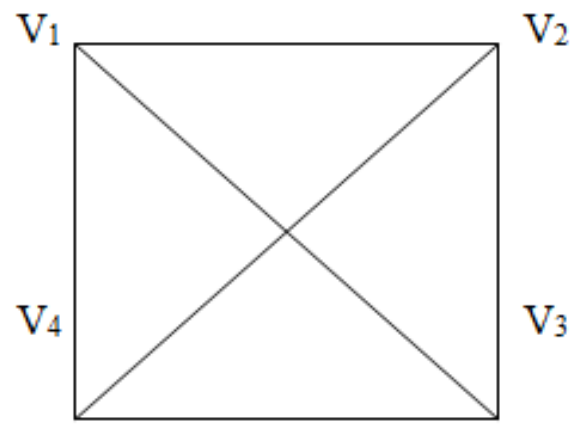

$\partial(\mathrm{G})=\Delta(\mathrm{G})=3$

It is a regular graph.

The calculated value for Mostar index is 0 .

It is a distance balanced graph.

\section{Example 2:}

Suppose we are removing one edge, we have

Removing an edge,

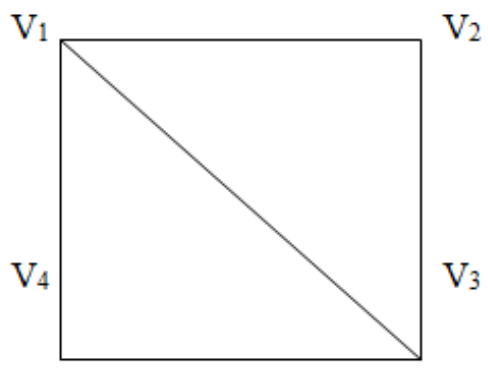

Here $\partial(\mathrm{G})=2$ and $\Delta(\mathrm{G})=3$

So, $\partial(\mathrm{G}) \neq \Delta(\mathrm{G})$

Hence it is not a regular graph,

So $\mathrm{M}_{\mathrm{o}}(\mathrm{G}) \neq 0$.

The calculated value for Mostar index of $G$ is 


$$
\mathrm{M}_{\mathrm{o}}(\mathrm{G})=1 .
$$

So it is not a distance balanced graph.

\section{Cubic Distance Balanced Graph}

A graph is said to be a rdb graph ( i.e regular distance balanced graph) if $\partial(G)=\Delta(G)$ and also $M o(G)=0$. Also it is a non $\operatorname{rdb}$ graph if $\partial(\mathrm{G}) \neq \Delta(\mathrm{G})$ and $\operatorname{Mo}(\mathrm{G}) \neq 0$

A graph is said to be cdb graph [cubic distance graph] if the mostar index of $\mathrm{G}$ is zero.

\section{Example:}

Let us consider the Petersen graph G,

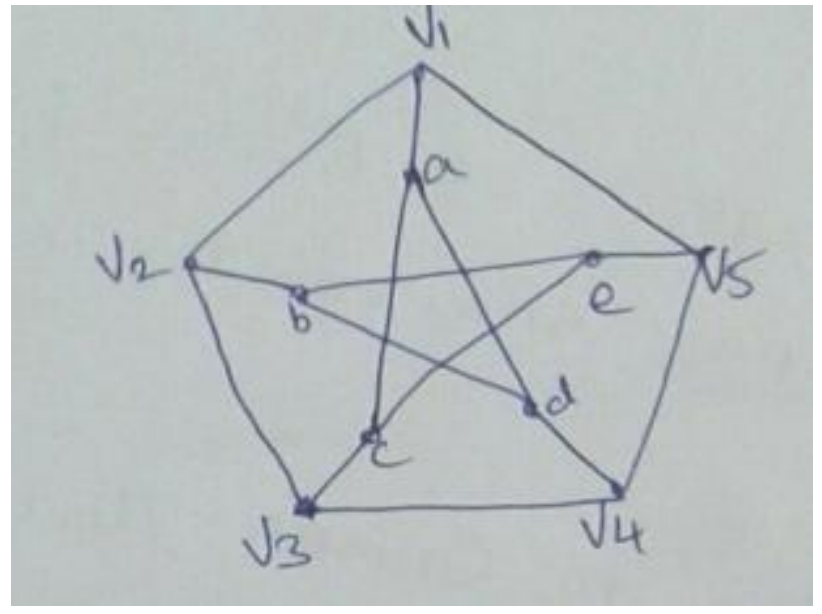

The Formula for mostar index of $\mathrm{G}$ is

$\mathrm{M}_{\mathrm{o}}(\mathrm{G})=\sum\left|\mathrm{n}_{\mathrm{u}}-\mathrm{n}_{\mathrm{v}}\right|$

The calculated value for mostar index of $\mathrm{G}$ is,

In edge $\mathrm{V}_{1} \mathrm{~V}_{5}$,

$\mathrm{u}->1+1+0+0+0+0+0+0$

$\mathrm{v}->0+0+1+0+0+1+0+0$

So $\left|\mathrm{n}_{\mathrm{u}}(\mathrm{e})-\mathrm{n}_{\mathrm{v}}(\mathrm{e})\right|=2-2=0$

In edge ad,

$\mathrm{u} \rightarrow>0+1+0+0+0+0+0+1$

$\mathrm{v}$-> $0+0+0+0+1+1+0+0$

$\left|\mathrm{n}_{\mathrm{u}}(\mathrm{e})-\mathrm{n}_{\mathrm{v}}(\mathrm{e})\right|=2-2=0$

In edge $\mathrm{V}_{2} \mathrm{~b}$

$\mathrm{u} \rightarrow>0+1+0+0+0+0+1+0$

$\mathrm{v}->0+0+1+0+1+0+0+0$

Clearly All $\sum \mathrm{V}_{\mathrm{i}} \mathrm{V}_{\mathrm{j}}=0$

$\mathrm{i} \neq \mathrm{j}$

So $\mathrm{M}_{\mathrm{o}}(\mathrm{G})=0$

Hence the cubic graph is also a distance balanced graph.

In edge $\mathrm{V}_{5} \mathrm{~V}_{6}$,

$\mathrm{u} \rightarrow>0+1+0+0+0+0+1+0$

$\mathrm{v}$-> $0+0+1+0+1+0+0+0$ 
$\left|\mathrm{n}_{\mathrm{u}}(\mathrm{e})-\mathrm{n}_{\mathrm{v}}(\mathrm{e})\right|=2-2=0$

II $^{\mathrm{ly}} \mathrm{All} \sum \mathrm{V}_{\mathrm{i}} \mathrm{V}_{\mathrm{j}}=0$

$\mathrm{i} \neq \mathrm{j}$

So $\mathrm{M}_{\mathrm{o}}(\mathrm{G})=0$

Hence the cubic graph is also a distance balanced graph.

\section{Theorem:}

Every cdbgraph [cubic distance graph] has an even number of points

\section{Proof:}

Let $\mathrm{G}$ be a cdb graph with $\mathrm{P}$ points.

Then $\sum \operatorname{deg} V_{i}=3 p$ which is even.

Hence every cdb graph has an even number of points.

\section{Conclusion:}

In this paper we have found the Mostar index of certain graphs are distance balanced graphs. Also we discussed the mostar index of certain regular and non-regular graphs. We proved these regular and non-regular graphs are distance balanced graphs. Mainly we have found that cubic graphs are also distance balanced graphs. In future studies we will briefly discuss about which of the graphs are distance balanced graphs.

\section{References}

J.Jerebic, S.Klavzar, F.Rall : Distance balanced graphs Ann.comb 12 (2008), 71-79

D.B.West, Introduction to Graph Theory, Prentice-Hall, Upper saddle River, NJ, 1996

H.Wiener, structural determination of the paraffin points, J.AM.chem Soc., 69 (1947) 17-20

S.Miklavic, P.Sparl : Distance balanced graphs. Discrete Appl Math 244(2018), 143-154

A.Ilic, S.Klazar, M.Milanovie: On distance balanced graphs, Eur. .Comb 31(2010), 733-737

D.B.West, Introduction to graph theory ( Prentice-Hall, upper saddle river,1996)

F.Harary, Graph theory (Addison-wesley, reading 1969)

I.Gutman, B.Furtula, distance in molecular graphs, univ.kragujevqc(2012)

I.Gutman, A formula for the wiener number of trees and its extension to graphs containing cycles. Graph theory notes New York XXVII, 9-15 (1994).

R.Sharadini, T Reti, on the transmission-based graph topological indices arxiv: 1710.08176V1 